\title{
INDUCED REPRESENTATIONS OF DISCRETE GROUPS
}

\section{LAWRENCE CORWIN ${ }^{1}$}

ABSTRACT. We determine necessary and sufficient conditions for a unitary representation of a discrete group induced from a finite-dimensional representation to be irreducible, and also briefly examine the question of when these induced representations are Type II.

Induced representations play an important role in the theory of representations of locally compact groups, and it is of some interest to know when an induced representation is irreducible. In the case where the representation $\pi$ on the separable locally compact group $G$ is induced from an irreducible representation $\sigma$ on a normal subgroup $H$, a simple criterion is known. Let $\sigma^{x}(x \in G)$ be the representation defined by the action of $x$ on $H: \sigma^{x}(h)=\sigma\left(x h x^{-1}\right)$. Then $\pi$ is irreducible $\Leftrightarrow \sigma^{x} \cong \sigma$ only if $x \in H$. (See [2] for a proof; $\Rightarrow$ is elementary, but $\Leftarrow$ requires direct integral theory.) In general, of course, anything so simple is too much to hope for. However, in the case where $G$ is discrete, one can hope that at least the question will have some sort of reasonable answer. In this case, one could ask as well for further information about $\pi$. In this paper, we find an irreducibility criterion for $\pi$ in the case where $\operatorname{dim} \sigma$ is finite, and say a bit (but only a bit) about other properties $\pi$ may have.

Given the subgroup $H$ of the discrete group $G$, we define the quasinormalizer $Q_{H}$ of $H$ to be $\left\{x \in G: x^{-1} H x \cap H\right.$ has finite index in $H$ and in $\left.x^{-1} H x\right\}$. For convenience, we denote $H \cap x^{-1} H x$ by $H_{x}$. (Then $x \in Q_{H}$ $\Leftrightarrow H_{x}$ and $H_{x-1}$ both have finite index in $H_{\text {.) }}$

Lemma 1. $H_{x}$ has finite index in $H \Leftrightarrow x H$ is contained in finitely many right cosets $H y$ of $H \Leftrightarrow H x H$ is a finite union of right cosets of $H$.

Proof. The second equivalence is straightforward, so we work on the first.

Received by the editors December 24, 1973.

AMS (MOS) subject classifications (1970). Primary 20E99, 22D10.

1Sloan Foundation Fellow. 
$\Rightarrow$ : if $\exists$ elements $x_{1}, \cdots, x_{n}$ with $H=\bigcup_{j=1}^{n} H_{x} x_{j}$, then let $H_{x}=$ $x^{-1} K x(K \subseteq H)$, and

$$
x H \subseteq \bigcup_{j=1}^{n} K x x_{j} \subseteq \bigcup_{j=1}^{n} H x x_{j}
$$

$\Leftarrow$ : It suffices to show that $x h_{1}$ and $x h_{2}$ are in the same right $H$-coset $\left(h_{j} \in H\right) \Leftrightarrow h_{1}, h_{2}$ are in the same right $H_{x}$ coset. If $x h_{1}, x h_{2} \in H y$, then $x h_{1} h_{2}^{-1} x^{-1} \in H$, or $h_{1} h_{2}^{-1} \in x^{-1} H x$; thus $h_{1} h_{2}^{-1} \in H_{x}$. Conversely, if $h_{1}, h_{2} \in H_{x} z$, then let $h_{j}=x^{-1} h_{j}^{\prime} x z\left(h_{j}^{\prime} \in H\right) ; x h_{j} \in h_{j}^{\prime} x z=H x z$, or $x h_{1}$ and $x h_{2}$ are in the same right coset.

We might note that $\left[H: H_{x-1}\right]$ can be infinite even if $\left[H: H_{x}\right]<\infty$. For instance, let $G$ be the free group on two generators $x, y$, and $H$ is generated by the elements $x^{j} y x^{-j}(j=1,2, \cdots)$, then $x^{-1} H x \supset H$ and $\left[x^{-1} H x: H\right]=\infty$. Hence $H_{x}=H$, but $\left[x^{-1} H x: H_{x}\right]=\infty$.

Lemma 2. If $K$ is a subgroup of $H$ of finite index, then $Q_{H}=Q_{K}$.

Proof. If $x \in Q_{K}$, then $K_{x}$ has finite index in $K$ and hence in $H$, but $K_{x} \subseteq H_{x}$. The same argument works with $H_{x-1}$. Hence $Q_{K} \subseteq Q_{H}$.

Conversely, $x \in Q_{H} \Rightarrow H_{x}$ has finite index in $H$; but then $x^{-1} H x \cap H$ $\cap K=x^{-1} H x \cap K$ has finite index in $H \cap K=K$, and similarly $x^{-1} K x \cap$ $K=K_{x}$ has finite index in $x^{-1} H x \cap K$. Combining these, $K_{x}$ has finite index in $K$; again, $K_{x-1}$ goes the same way.

Theorem 1. $Q_{H}$ is a group containing $H$.

Proof. Since $x \in Q_{H} \Leftrightarrow H_{x}, H_{x-1}$ have finite index in $H, Q_{H}$ is closed under inverses. That $H \subseteq Q_{H}$ is clear.

If $x, y \in Q_{H}$, then, for instance, $H_{x}$ is of finite index in $H$; from Lemma 2, $y^{-1}\left(x^{-1} H x \cap H\right) y^{-1} \cap H_{x}$ is of finite index in $H_{x}$. This shows that $H_{x y}$ is of finite index in $H$. The other half is similar.

Now let $\sigma$ be a finite-dimensional irreducible representation of $H$ on a complex inner product space $\mathcal{H}_{0}$, and let $\pi$ be the corresponding induced representation of $G$ (on $\mathcal{H}$, say). We denote the algebra of intertwining operators of $\pi$ with itself by $\operatorname{Hom}(\pi, \pi)$. If $A \in \operatorname{Hom}(\pi, \pi)$, then we can describe $A$ as follows: let $\delta_{e}^{(v)}$ be the function which is $\sigma(h) v$ at $h \in H$ and 0 off $H$. Then $v \mapsto\left(A \delta_{e}^{(v)}\right)(x)$ is a linear operator on $\mathcal{H}_{0}$, which we denote by $A_{x}$.

Lemma 3. The $A_{x}$ determine $A$. 
Proof. Let $\delta_{y}^{(v)}$ be $\sigma(h) v$ at hy and 0 off $H y$. Then $\delta_{y}^{(v)}=\pi\left(y^{-1}\right)$. $\delta_{e}^{(v)}$, and therefore

$$
\left(A \delta_{y}^{(v)}(x)\right)=A \pi\left(y^{-1}\right) \delta_{e}^{(v)}(x)=\pi\left(y^{-1}\right)\left(A \delta_{e}^{(v)}\right)(x)=A \delta_{e}^{(v)}\left(x y^{-1}\right),
$$

or

$$
A \delta_{y}^{(v)}(x)=A_{x y}-1
$$

Hence the $A_{x}$ determine $A$ on a dense subset of $\mathcal{H}$, and this proves the lemma.

Note. We also have (from (1))

$$
A \delta_{y}^{(v)}=\sum_{x \in H / G} \delta_{x}^{(w)}, \text { where } w=A_{x y}-1
$$

For the next theorem, we need the following combinatorial lemma.

Lemma 4. A group $G$ cannot be covered by finitely many cosets of finitely many subgroups of infinite index.

Proof. We may assume that all cosets are right cosets, since $x H=$ $\left(x H x^{-1}\right) x$. We use induction on the number $n$ of subgroups involved. Assume the theorem for $n$, and suppose that the cosets are cosets of $H_{1}, \cdots$, $H_{n+1}$. Let $K_{j}=K_{j} \cap H_{n+1}$. We may assume that if $1 \leq j \leq n, H_{j} \cap H_{n+1}$ has infinite index in $H_{n+1}$; otherwise, $H_{n+1}$ is covered by finitely many cosets of $H_{j}$, and therefore we can replace $H_{n+1}$-cosets with $H_{j}$-cosets. We may also assume (perhaps by translating all cosets by some $x \in G$ ) that $H_{n+1}$ itself is not one of the cosets in the union. Hence $H_{n+1}$ is covered by finitely many cosets of $H_{1}, \cdots, H_{n}$, and therefore $H_{n+1}$ is the union of finitely many cosets of $K_{1}, \cdots, K_{n}$. The inductive hypothesis says that this is impossible, and the lemma follows.

Theorem 2. If $A=\left\{A_{x}\right\} \in \operatorname{Hom}(\pi, \pi)$, then $A_{x}=0$ unless $x \in Q_{H}$. For $x \in Q_{H}$ and $h, h^{\prime} \in H$, we have $A_{h \times h^{\prime}}=\sigma(h) A_{x} \sigma\left(h^{\prime}\right)$, and $A_{x}$ intertwines $\sigma$ and $\sigma^{x}\left(\right.$ where $\left.\sigma^{x}\left(x^{-1} h x\right)=\sigma(h)\right)$ on $H_{x}$.

Proof. $A_{h x}^{(v)}=A \delta_{e}^{(v)}(h x)=\sigma(h) A \delta_{e}^{(v)}(x)=\sigma(h) A_{x}(v)$, because $A \delta_{e}^{(v)} \epsilon$ $\mathcal{H}$. Furthermore, if $h \in H, \delta_{h}^{(v)}$ and $\delta^{(w)}$, where $w=\sigma\left(h^{-1}\right) v$, are both concentrated on $H$ and agree at $h$; thus they are equal. But

$$
A \delta_{h}^{(v)}(x)=A_{x h^{-1}}(v) \text { and } A \delta_{e}^{(w)}(x)=A_{x} \sigma\left(h^{-1}\right) v
$$

Thus $A_{h x}=\sigma(h) A_{x}$ and $A_{x h^{\prime}}=A_{x} \sigma\left(h^{\prime}\right)$ for $h, h^{\prime} \in H$. If $h=x^{-1} h_{0} x$ $\left(h, h_{0} \in H\right)$, then 


$$
\sigma^{x}(b) A_{x}=\sigma\left(h_{0}\right) A_{x}=A_{h_{0} x}=A_{x h}=A_{x} \sigma(h),
$$

which proves the claim about $A_{x}$ being an intertwining operator. So all that we need to show is that if $A_{x} \neq 0$, then $x \in Q_{H}$.

Assume $A_{x} \neq 0$. For each unit vector $v \in \mathcal{H}_{0}$, there is an element $h \in H$ such that $A_{x} \sigma(h) v \neq 0$, since the unit vectors $\sigma(h) v$ span $\mathcal{H}_{0}$. Thus the open subsets $U_{h}=\left\{v:\|v\|=1, A_{x} \sigma(h) v \neq 0\right\}$ cover the unit ball of $\mathcal{H}$. We may pick a finite set $\left\{h_{1}, \cdots, h_{n}\right\} \subseteq H$, plus a number $\alpha>0$, such that for any unit vector $v$, some vector $A_{x} \sigma\left(h_{i}\right) v$ has length $\geq a$. Now suppose that $\left[H: H_{x}\right]=\infty$. Then we can pick elements $k_{1}, k_{2}, \cdots$ of $H$ such that if $i \neq j$, the cosets $H_{x} b_{1} k_{i}, \cdots, H_{x} h_{n} k_{i}$ are distinct from the cosets $H_{x} b_{1} k_{j}, \cdots, H_{x} b_{n} k_{j}$. (This follows from the fact that a group cannot be covered by finitely many cosets of subgroups of infinite index.) We know that for each $k_{i}$, one of the vectors $A_{x} \sigma\left(b_{1} k_{i}\right) v, \cdots, A_{x} \sigma\left(h_{n} k_{i}\right) v$ has length $\geq a$. Let it be $A_{x} \sigma\left(h_{j(i)} k_{i}\right) v$. As the elements $x h_{j(i)} k_{i}$ are in distinct right $H$-cosets,

$$
\left\|A \delta_{e}^{(v)}\right\| \geq \sum_{i=1}^{\infty}\left\|A_{x h_{j(i)}} k_{i} v\right\| \geq \sum_{i=1}^{\infty} \alpha^{2}=\infty,
$$

which is impossible. Hence $\left[H: H_{x}\right]<\infty$.

Finally, we compute

$$
\begin{aligned}
A^{*}:\left\langle\delta_{x}^{(w)}, A^{*} \delta_{e}^{(v)}\right\rangle & =\left\langle A \delta_{x}^{(w)}, \delta_{e}\right\rangle=\left\langle A \delta_{x}^{(w)}(e), v\right\rangle \\
& =\left\langle A_{x^{-1}} w, v\right\rangle=\left\langle w,\left(A_{x^{-1}}\right)^{*} v\right\rangle=\left\langle\delta_{x}^{(w)}, B \delta_{e}^{(v)}\right\rangle,
\end{aligned}
$$

where $B_{x}=\left(A_{x-1}\right)^{*}$. Hence

$$
\left(A^{*}\right)_{x}=\left(A_{x^{-1}}\right)^{*}
$$

But $A^{*} \in \operatorname{Hom}(\pi, \pi)$; hence $\left[H: H_{x-1}\right]<\infty$. This completes the proof. Note, incidentally, that $A_{x}$ also intertwines $\sigma^{x-1}$ and $\sigma$ on $H_{x-1}$.

We now prove a converse of sorts to Theorem 2. First, a lemma.

Lemma 5. Let $\left\{T_{j}\right\}_{j=1}^{m}$ be any sequence of operators on a finite-dimensional inner product space $V$. There is a constant $c$ (depending only on $\operatorname{dim} V$ ) and a vector $v \in V$ such that

$$
c \sum_{j=1}^{m}\left\|T_{j} v\right\|^{2} \geq \sum_{j=1}^{m}\left\|T_{j}\right\|^{2} .
$$

Proof. Let $v_{1}, \cdots, v_{n}$ be an orthonormal basis for $V$, and let \|\|$\|$ be the trace norm on operators $\left(\|T\|^{2}=\operatorname{Tr}(T * T)\right)$. As all norms on $\mathcal{B}(V)$ are equivalent, and as 


$$
\sum_{i=1}^{n}\left\|T_{j} v_{i}\right\|^{2}=\sum_{i=1}^{n}\left\langle T_{j}^{*} T_{j} v_{i}, v_{i}\right\rangle=\left\|T_{j}\right\|^{2}
$$

there is a constant $c_{0}$ such that $c_{0} \Sigma_{i=1}^{n}\left\|T_{j} v_{i}\right\|^{2} \geq\left\|T_{j}\right\|^{2}$ for all $j\left(c_{0}\right.$ depends only on $n=\operatorname{dim} V$ ). Summing, we see that

$$
c_{0} \sum_{j=1}^{m} \sum_{i=1}^{n}\left\|T_{j} v_{i}\right\|^{2} \geq \sum_{j=1}^{m}\left\|T_{j}\right\|^{2},
$$

and hence

$$
\exists i: c_{0} n \sum_{j=1}^{m}\left\|T_{j} v_{i}\right\|^{2} \geq \sum_{j=1}^{m}\left\|T_{j}\right\|^{2}
$$

Let $c=c_{0} n$.

Theorem 3. Suppose that $x \in Q_{H}$, and that $A_{x}$ intertwines $\sigma$ and $\sigma^{x}$ on $H_{x}$. Define $A=\left\{A_{y}\right\}$, where $A y=\sigma(h) A_{x} \sigma\left(h^{\prime}\right)$ if $y=h x h^{\prime}$ and 0 otherwise. Then $A \in \operatorname{Hom}(\pi, \pi)$. The $A$ 's span a weakly dense subspace in $\operatorname{Hom}(\pi, \pi)$.

\section{Proof. Let}

$$
\left(A^{0} f\right)(y)=\sum_{b \in H} \sigma\left(h^{-1}\right) A_{x} f\left(x^{-1} h y\right) .
$$

The sum is finite and well defined; if we replace $h$ by $h_{1} h$, where $h_{1} \in H_{x}$ (so that $h_{1} \in H, h_{1}=x h_{2} x^{-1}$ with $h_{2} \in H$ ), then

$$
\begin{aligned}
\sigma\left(h_{1} h\right)^{-1} A_{x} f\left(x^{-1} h_{1} h y\right) & =\sigma\left(b^{-1}\right) \sigma\left(h_{1}^{-1}\right) A_{x} f\left(h_{2} x^{-1} b y\right) \\
& =\sigma\left(b^{-1} \sigma\left(b^{-1}\right) A_{x} \sigma\left(h_{2}\right) f\left(x^{-1} b y\right)\right. \\
& =\sigma\left(b^{-1}\right) \sigma\left(b_{1}^{-1}\right) \sigma^{x}\left(h_{2}\right) A_{x} f\left(x^{-1} b y\right) \\
& =\sigma\left(b^{-1}\right) A_{x} f\left(x^{-1} h y\right),
\end{aligned}
$$

so that each term is independent of the coset representative taken. Also, if $h^{\prime} \in H$,

$$
\begin{aligned}
\left(A^{0} f\right)\left(h^{\prime} y\right) & =\sum_{h} \sigma\left(h^{-1}\right) A_{x} f\left(x^{-1} h h^{\prime} y\right) \\
& =\left(h \mapsto b h^{\prime-1}\right) \sum_{h} \sigma\left(h^{\prime}\right) \sigma\left(b^{-1}\right) f\left(x^{-1} h y\right)=\sigma\left(h^{\prime}\right) A^{0} f(y) .
\end{aligned}
$$

Hence $A^{0}: \mathcal{H} \rightarrow \mathcal{H}$. Clearly $A^{0} \in \operatorname{Hom}(\pi, \pi)$. Finally, we compute $\left(A^{0}\right) y$. Since

$$
A_{y}^{0}(v)=\sum_{h} \sigma(h) A_{x} \delta_{e}^{(v)}\left(x^{-1} h y\right)
$$


from (1), and since this sum is 0 unless $x^{-1} b y \in H$ for some $b \in H, A_{y}^{0}=0$ for $y \notin H x H$. If $y=h_{1} x h_{2}$, then we may assume that $h_{1}$ is one of the coset representatives for $\mathrm{Hx}^{-1} H$; the term involving $h_{1}$ will be the only nonzero one, and

$$
A_{h_{1} \times h_{2}}^{0}(v)=\sigma\left(h_{1}\right) A_{x} \delta_{e}^{(v)}\left(h_{2}\right)=\sigma\left(h_{1}\right) A_{x} \sigma\left(h_{2}\right) v .
$$

Hence $A^{0}=A$.

Now let $B=\left\{B_{y}\right\}$ be an arbitrary element of $\operatorname{Hom}(\pi, \pi)$. Then for every $v \in \mathcal{H}_{0}$ of length $1,\left\|B \delta_{e}^{(v)}\right\| \leq\|B\|$; hence $\Sigma_{y \in H \backslash G}\left\|B_{y}(v)\right\|^{2} \leq\|B\|^{2}$. Lemma 5 says that $\Sigma_{y \in H \backslash G}\left\|B_{y}\right\|^{2}<\infty$. In particular, $B_{y}$ is concentrated on countably many right $H$-cosets of $G$. If

Now let

$$
f=\sum_{j=1}^{n} \alpha_{j} \delta_{x_{j}}^{\left(v_{j}\right)} \text {, then }(B f)(z)=\sum_{j=1}^{n} a_{j} B_{z x_{j}^{-1}}\left(v_{j}\right) \text {. }
$$

$$
f=\sum_{j=1}^{\infty} \alpha_{j} \delta_{x_{j}}^{\left(v_{j}\right)} \in L^{2}(\mathcal{H})(G), \quad f_{n}=\sum_{j=1}^{n} a_{j} \delta_{x_{j}}^{\left(v_{j}\right)} .
$$

Because $f_{n} \rightarrow f, B f_{n} \rightarrow B f$; hence $\sum_{j=1}^{\infty} \alpha_{j} B_{z x_{j}^{-1}}\left(v_{j}\right)$ converges. If we pick a basis of orthonormal vectors in $V$, we see similarly that the sum converges for each component. Moreover, it converges for each component regardless of how the terms are rearranged, since we can label the $x_{j}$ in any order. Thus the sum for each component converges absolutely. Now order the double cosets $H y_{j} H$ on which $B_{y}$ is nonzero, and let $B_{y}^{(n)}=B_{y}$ if $y \epsilon$ $\bigcup_{j=1}^{n} H y{ }_{j} H$, and 0 otherwise. Then $B_{y}^{(n)}$ is in the space spanned by the $A^{\prime}$ 's, and if $f=\Sigma_{j=1}^{\infty} \alpha_{j} \delta_{x j}^{\left(v_{j}\right)}$,

$$
\left(B^{(n)} f\right)(z)=\sum_{j=1}^{\infty} a_{j} B_{z x_{j}^{-1}}^{(n)}\left(v_{j}\right) \rightarrow B f(z) \text { as } n \rightarrow \infty .
$$

Hence $B^{(n)} \rightarrow B$ strongly (a fortiori weakly).

Corollary 1. $\pi$ is irreducible $\Leftrightarrow$ for all $x \in Q_{H^{\prime}}, x \in H, \pi^{(x)}$ and $\pi$ are disjoint on $H_{x}$.

Corollary 2. Let $\tau=$ Ind $\sigma_{H \rightarrow Q_{H}}$. Then $\operatorname{Hom}(\pi, \pi) \cong \operatorname{Hom}(\tau, \tau)$.

As an example, let $G$ be the free group on two generators $x$ and $y$, and let $H$ be the subgroup generated by $x$. Then $Q_{H}=H$, and it follows, for instance, that the "regular" representation of $G$ on $H \backslash G$ is irreducible.

If $G$ is finite (so that $Q_{H}=G$ ), Corollary 1 amounts to Mackey's generalization of a theorem of Shoda [ 1 , Theorem 6]. 
Next, we look briefly at the center of $\operatorname{Hom}(\pi, \pi)$. We shall assume (as we may without loss of generality) that $G=Q_{H}$.

Theorem 4. $B=\left\{B_{y}\right\}$ is central in $\operatorname{Hom}(\pi, \pi) \Leftrightarrow$ for all $x, z \in G$ and all $A_{x}$ intertwining $\sigma$ and $\sigma^{*}$ on $H_{x}$,

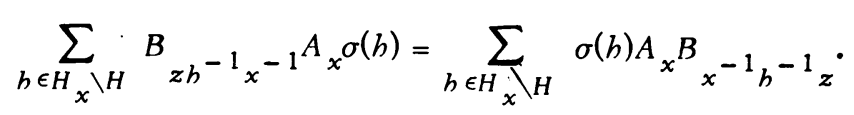

Proof. Let $A=\left\{A_{y}\right\}$ be the operator constructed from $A_{x}$ as in Theorem 3. We know that $B$ is central $\Leftrightarrow A B=B A$, all such $A \Leftrightarrow A B \delta_{e}^{(\nu)}$ $=B A \delta_{e}^{(v)}$, all such $A$. So we compute

$$
B A \delta_{e}^{(v)}(y)=B\left(\sum_{y \in H \backslash H x M} \delta_{y}^{\left(w_{y}\right)}\right)(z)=\sum_{y \in H \backslash H x H} B z_{z y^{-1}} A_{y}(v) \quad\left(w_{y}=A_{y}(v)\right) .
$$

But to get the coset representatives, we may let $y=x h, h$ running through coset representatives of $H_{x} \backslash H$; thus

$$
\begin{aligned}
& B A \delta_{e}^{(v)}(z)=\sum_{b \in H_{x} \backslash H} B b^{-1} x^{-1} A_{x h}(v)=\sum_{b \in H_{x} \backslash H} B b^{-1} x^{-1} A_{x} \sigma(h) v, \\
& A B \delta_{e}^{(v)}(z)=A\left(\sum_{y \in H \backslash G} \delta_{y}^{\left(v_{y}\right)}(z)\right)=\sum_{y \in H \backslash G} A_{z y}{ }^{-1} B_{y}(v) \quad\left(v_{y}=B_{y}(v)\right) .
\end{aligned}
$$

Again, we must have $z y^{-1}=h x h^{\prime}$, or $y=h^{\prime-1} x^{-1} h^{-1} z$; to get coset representatives, let $h^{\prime}=e$ and $h$ run through $H_{x} \backslash H$. One gets

$$
A B \delta_{e}^{(v)}(z)=\sum_{h \in H_{x} \backslash H} A_{h x} B_{x^{-1} h^{-1} z}(v)=\sum_{b \in H_{x} \backslash H} \sigma(h) A_{x} B_{x^{-1} b^{-1} z}(v) .
$$

Now combine (4) and (5).

Corollary 1. If $x$ normalizes $H$ and $\sigma=\sigma^{x}$ on $H$, then one has

if $B$ is central.

$$
A_{x}^{-1} B_{z} A_{x}=B_{x}^{-1} z
$$

Corollary 2. If $\operatorname{dim} \mathcal{H}_{0}=1$, the condition of Theorem 4 becomes

$$
\sum_{h \in H_{x} H} \sigma(h) B z_{z h^{-1} x^{-1}}=\sum_{h \in H_{x} \backslash H} \sigma(h) B x_{x^{-1} h^{-1} z}
$$

These sums are unpleasant to deal with; in one case, where they disappear, we can say a little.

Theorem 5. Let $N_{H}=\left\{x \in G: x\right.$ normalizes $H$ and $\left.\sigma^{x}=\sigma\right\}, M_{H}=$ 
$\left\{x \in N_{H}\right.$ : the $N_{H}$-conjugates of $x$ lie in finitely many $H$-cosets $\}$. If $\left[N_{H}=\right.$ $\left.M_{H}\right]=\infty, \pi$ is Type II.

Proof. We may assume $G=N_{H}$ without loss of generality; in particular, $H$ is normal. From this, it follows easily that $M_{H}$ is indeed a group. Now one can mimic Mautner's proof in [3] that if $G$ is a discrete group, $G_{0}$ is the subgroup of elements with finitely many conjugates, and $\left[G: G_{0}\right]$ is infinite, then the regular representation of $G$ is Type II. This proves the theorem.

Theorem 6. In the above situation, let $M^{\prime}{ }_{H}=$ commutator subgroup of $M_{H}$. If $\left[M^{\prime}{ }_{H}: H \cap M_{H}^{\prime}\right]$ is infinite, $\pi$ is Type II.

Proof. From Theorem 5, we may as well assume $\left[N_{H}: M_{H}\right]<\infty$; then we may as well assume that $N_{H}=M_{H}$, since inducing from $M_{H}$ to $N_{H}$ will change nothing. Suppose that $\pi$ has a Type $\mathrm{I}_{n}$ factor for some $n \geq 1$. The argument in Theorem 1 of [4] now goes through essentially unchanged to show that the commutator subgroup $\left[M_{H} / H, M_{H} / H\right]$ is finite, and this implies the result.

Theorem 7. Suppose $G=N_{H}$ and $\pi$ is Type II. Then $\left[N_{H}=M_{H}\right]=\infty$ or $\left[M_{H}^{\prime}: H \cap M_{H}^{\prime}\right]$ is infinite.

Proof. Pick $x \in G$, and let $A_{x}$ implement the equivalence of $\sigma$ with $\sigma^{(x)}$. Let $C_{1}$ project $H_{\text {onto }} \mathcal{H}_{0}$ by evaluation at $x_{1}$ and $C_{0}$ by evaluation at $e$. Then $C_{0} \in \operatorname{Hom}(\pi / H, \sigma)$, and $C_{1} \in \operatorname{Hom}\left(\pi \mid H, \sigma^{x}\right)$, since

$$
\begin{aligned}
\pi(h) \delta_{x}^{(v)}(x) & =\delta_{x}^{(v)}(x h)=\delta_{x}^{(v)}\left(x h x^{-1} \cdot x\right) \\
& =\sigma\left(x h x^{-1}\right)\left(\delta_{x}^{(v)}(x)\right)=\sigma^{(x)}(h)\left(\delta_{x}^{(v)}(x)\right) .
\end{aligned}
$$

Thus $A_{x} C_{0}$ is a multiple of $C_{2}$, and we may change $A_{x}$ if necessary so that $A_{x} C_{0}=C_{1}$. Note that if we change $x$ to $h x$, the correct choice for $A_{h x}$ is $\sigma(h) A_{x}$.

Now let $P$ be the operator given by

$$
P(f) y=\frac{1}{m} \sum_{x \in M \backslash H M_{M}} A_{x^{-1}} f(x y),
$$

where $m=\left[M_{H}^{\prime}: H\right] . P \in \operatorname{Hom}(\pi, \pi)$; in fact, $P=\left(P_{x}\right)$, where $P_{x}=A_{x} / m$ for $x \in M_{H}^{\prime} H$, and $=0$ otherwise. Since $A_{x} A_{y}=A_{x y}$ (thanks to our normalization), $P^{2}=P$, and Theorem 4 implies that $P$ is central. Now the last part of the proof of Theorem 1 of [4] goes through (with $P$ for $f$ ) without any trouble. 
Theorems 5, 6, and 7 are more or less copies of the corresponding results for $H=\{e\}$. In case $H$ is not normal, the situation seems considerably more obscure.

Added in proof. Lemma 4 is found in B. H. Neumann, Groups covered by permutable subsets, J. London Math. Soc. 29 (1954), 236-248. MR 15, 931. Corollary 1 was proved by Mackey in the case where $\operatorname{dim} \sigma=1$. See [1].

\section{BIBLIOGR APHY}

1. G. W. Mackey, On induced representations of groups, Amer. J. Math. 73 (1951), 576-592. MR 13, 106.

2. - Unitary representations of group extensions. I, Acta Math. 99 (1958), 265-311. MR 20 \#489.

3. F. Mautner, The structure of the regular representation of certain discrete groups, Duke Math. J. 17 (1950), 437-441. MR 12, 588.

4. M. Smith, Regular representations of discrete groups, J. Functional Analysis 11 (1971), 401-406.

SCHOOL OF MATHEMATICS, THE INSTITUTE FOR ADVANCED STUDY, PRINCETON, NEW JERSEY 08540

Current address: Department of Mathematics, Yale University, New Haven, Connecticut 06520 